\title{
ANALISE DE RISCO EM RIOS NATURAIS SUJEITOS A LANÇAMENTOS DIFUSOS DE POLUENTES
}

\author{
Raque Jucá de Moraes Sales ${ }^{1}$
}

Juliana Alencar Firmo de Araújo ${ }^{2}$

Patrícia Freire Chagas $^{3}$

\begin{abstract}
RESUMO
O risco de um possível desequilíbrio entre oferta e demanda de água tem contribuído com significativos avanços para a Engenharia dos Recursos Hídricos. Associando este fato aos novos conceitos de sustentabilidade ambiental, percebe-se a necessidade do estabelecimento e aplicação de medidas rigorosas de controle do uso de rios, lagos, estuários e oceanos. A utilização de novas teorias, juntamente com o desenvolvimento de modelos matemáticos representativos, permite novas perspectivas no entendimento dos complexos processos inerentes a esta área do conhecimento. Nesta pesquisa, foi desenvolvida uma metodologia que combina a Teoria Fuzzy com processos de transporte de poluente, objetivando avaliar o risco de um rio natural sofrer processo de degradação ambiental quando sujeito a lançamentos difusos. A metodologia emprega fundamentos da Teoria Fuzzy para avaliar a solução da equação da difusão advectiva em uma estrutura fuzzy e, com isto, transformar um campo de concentração, variável no tempo e espaço, em um campo de funções de pertinência, também variável no tempo e espaço. Este processo foi obtido através do uso de dados fuzzy nos modelos determinísticos. A partir daí torna-se possível incorporar aspectos de incertezas no modelo matemático proposto e avaliar o risco de degradação ambiental em rios sujeitos a lançamentos difusos.
\end{abstract}

PALAVRAS-CHAVE: Risco. Teoria Fuzzy. Modelagem matemática.

\section{RISK ANALYSIS IN NATURAL RIVERS RELEASES SUBJECT TO DIFFUSE POLLUTANT DISCHARGE}

\section{ABSTRACT}

The risks of a possible unbalance between offer and demand of water, both of them, at national or international level, have been contributing with significant progresses to the Water Resources Engineering. Associating to this fact the new concepts of environmental sustainability, there is the necessity of the establishment and the

\footnotetext{
${ }^{1}$ Doutora em Recursos Hídricos pela Universidade Federal do Ceará. Bolsista PNPD-CAPES. raqueljuca@gmail.com.

${ }^{2}$ Mestre e doutoranda em Recursos Hídricos pela Universidade Federal do Ceará. Bolsista CAPES. judiaraujo@yahoo.com.br;

${ }^{3}$ Doutora em Recursos Hídricos pela Universidade Federal do Ceará. Bolsista PNPD-CAPES. pfchagas@yahoo.com.
} 
application of more rigorous processes of control of the use of rivers, lakes, estuaries and oceans. The use of new theories, together with the development of mathematical models, more and more representative, has been allowing, to the researchers, new perspectives in the understanding of the complex processes inherent to this area of the knowledge. In this work, it was developed a methodology that combines the fuzzy theory with the pollutant transport processes, with the objective of evaluating the risk of a natural river to suffer a process of environmental degradation. The methodology uses the fuzzy theory to get the solution of the diffusion and advective equation, in a fuzzy structure and, in such way, to transform a concentration field, variable in the time and space, in a field of concentration membership functions, also variables in time and in space. This process was obtained through the application of the fuzzy data into the deterministic models. After the attainment of those functions, it is possible to incorporate the uncertainties aspects in the proposed mathematical model and, after that, do analyze the environmental degradation risk, in the river, that receives pollutant diffusion discharge.

KEY-WORDS: Risk. Fuzzy theory. mathematical modelling

\title{
ANÁLISIS DE RIESGOS EN LOS RÍOS NATURALES QUE RECIBE LA CONTAMINACIÓN DIFUSA
}

\begin{abstract}
RESUMEN
Los riesgos de un posible desequilibrio entre la oferta y demanda de agua, los dos de ellos, a nacional o el nivel internacional, han estado contribuyendo con los progresos significantes a la Ingeniería de Recursos de Agua. Asociando a este hecho los nuevos conceptos de mantenimiento medioambiental, ahí la necesidad del establecimiento y la aplicación de procesos más rigurosos de mando del uso de ríos, lagos, estuarios y océanos. El uso de nuevas teorías, junto con el desarrollo de modelos matemáticos, cada vez más representante, ha estado permitiendo, a los investigadores, nuevas perspectivas en la comprensión de los procesos complejos inherente a este área del conocimiento. En este trabajo, se desarrolló una metodología que combina la teoría rizada con los procesos de transporte de contaminante, con el objetivo de evaluar el riesgo de un río natural para sufrir un proceso de degradación medioambiental La metodología emplea fundamentos de la Teoría de aproximación para evaluar la solución de la ecuación de difusión advectivo en una estructura difusa y, por lo tanto, se convierten en un campo de concentración, variable en el espacio y el tiempo en un campo de funciones de pertenencia, también es variable en el tiempo y espacio.. Este proceso se obtuvo a través de la aplicación de los datos rizados en los modelos determinista. Después del logro de esas funciones, es posible incorporar los aspectos de incertidumbres en el modelo matemático propuesto y, después de eso, analice el riesgo de degradación medioambiental, en el río que recibe la descarga de la contaminación difusa.
\end{abstract}

PALABRAS-CLAVE Riesgo. Teoría Difusa. Los modelos matemáticos

\section{INTRODUÇÃO}

A possibilidade concreta de escassez de água doce se tornar, cada vez mais, uma ameaça ao desenvolvimento econômico e social no planeta. $O$ aumento populacional, a instalação de indústrias e o uso intenso do solo para fins agrícolas têm acelerado o acréscimo da demanda de água.

Atualmente, modelos matemáticos de qualidade de água têm sido utilizados 
para representar processos físicos através da formulação de um conjunto de equações matemáticas $e$, incorporado às equações, parâmetros com certo grau de incerteza. Estes procedimentos permitem avaliar a aleatoriedade ou indeterminação que podem estar relacionadas com os dados, medições dos parâmetros, métodos de análise, parâmetros hidráulicos e hidrológicos e formulações e aproximações nas soluções.

Muitas técnicas têm sido desenvolvidas com o objetivo de quantificar riscos em diversos problemas hídricos. Dentre as importantes teorias no trato deste problema, podem-se destacar a Teoria Probabilística e a Teoria Fuzzy. A primeira, bastante utilizada em diferentes campos da ciência, baseia-se nos princípios dos métodos probabilísticos para quantificar risco mediante avaliação de incertezas. A segunda exige, como fundamentação, o desenvolvimento de funções especiais, com propriedades intrínsecas à referida teoria, para avaliar risco (CHAGAS, 2005).

Entendendo que o estudo das incertezas é fundamental para o gerenciamento dos riscos nas questões de qualidade de água, esta pesquisa busca aplicar a Teoria Fuzzy em modelos advectivo-difusivos para avaliar o risco dos sistemas hídricos não apresentarem padrões de qualidade compatíveis com os usos desejados, quando sujeitos a lançamentos de poluentes. Deseja-se, enfim, fornecer subsídios que auxiliem a tomada de decisão nos mais diversos Programas de Conservação de Mananciais.

\section{METODOLOGIA}

O estudo se desenvolve mediante a combinação de um conjunto de teorias, aplicadas convenientemente, de modo que os objetivos propostos sejam alcançados. Para tanto, a metodologia terá por base a análise do comportamento do campo de concentração em um rio. Este campo de concentração é obtido a partir da solução da equação diferencial da difusão advectiva na forma fuzzy. Neste processo, um esquema numérico implícito, com base no método das diferenças finitas, é utilizado. O campo de concentração serve para avaliar possíveis riscos de contaminação deste 
corpo hídrico. Desta forma, o modelo em questão é apresentado na forma (JAMES, 1993):

$\frac{\partial C}{\partial t}+u \frac{\partial C}{\partial x}=\frac{1}{A} \frac{\partial}{\partial x}\left(A E \frac{\partial C}{\partial x}\right)-K C+S_{D}$

$C$ é a concentração da substância ao longo do canal, em $\mathrm{kg} / \mathrm{m}^{3}$; $u$ é a velocidade ao longo do canal, em $\mathrm{m} / \mathrm{s} ; A$ é a área da seção transversal do canal, em $\mathrm{m}^{2}$; $E$ é o coeficiente de dispersão longitudinal; $K$ é o coeficiente de decaimento da substância, em $\mathrm{T}^{-1} ; S_{D}$ representa o lançamento distribuído ao longo do canal.

Condições de contorno, equação 1, e condições iniciais, equação 2.

$\mathrm{C}(0, \mathrm{t})=\mathrm{C}(\mathrm{t}) ; \quad \frac{\partial C}{\partial x}(L, t)=0$

$\mathrm{C}(\mathrm{x}, 0)=\mathrm{C}(\mathrm{x})$

\subsection{Análise do Risco}

Para o estudo do Risco, foram empregados na pesquisa os princípios dos conjuntos difusos. O conceito central da Teoria Fuzzy é a definição das funções de pertinência, que representam numericamente o grau na qual um elemento pertence a um conjunto. No caso da teoria dos conjuntos clássicos, o valor da função de pertinência de cada elemento em um conjunto clássico é 1 para membros (aqueles que certamente pertencem) ou 0 para não membros (aqueles que não pertencem). Já na Teoria Fuzzy, verifica-se que com o aumento do grau de pertinência de um elemento em um conjunto, o valor da função de pertinência para esse elemento também aumenta dentro do intervalo [0,1] (BOGARDI; DUCKSTEIN, 2002). Num conjunto fuzzy $\tilde{X}$, para cada elemento $\mathrm{x}$, pertencente ao conjunto, existe uma função de pertinência associada:

$$
\widetilde{X}=\left\{\left(x, \mu_{\widetilde{X}}(x)\right) / x \in X\right\}
$$

$\mu_{\widetilde{X}}(x)$ é chamada função de pertinência ou grau de pertinência, h, de $\mathrm{x}$ em $\widetilde{X}$.

Como o valor máximo da função de pertinência é $h=1$, o conjunto é dito normal ou normalizado. Por exemplo, o conjunto fuzzy de mulheres muito altas compreenderia mulheres com altura de 1,80 metros, com nível de pertinência $h=1,0$; mulheres com altura de 1,75 metros, com nível de pertinência $h=0,5$; mulheres com altura de 1,70 metros, com nível de pertinência $h=0,1$. 
Para desenvolver a modelagem fuzzy há a necessidade de reescrever a equação do transporte de massa na sua forma fuzzy. Desta maneira, têm-se a equação do transporte de massa:

$$
\frac{\partial \widetilde{C}}{\partial t}+\widetilde{u} \frac{\partial \widetilde{C}}{\partial x}=\frac{1}{\widetilde{A}} \frac{\partial}{\partial x}\left(\widetilde{E} \widetilde{A} \frac{\partial \widetilde{C}}{\partial x}\right) \pm \widetilde{K} \widetilde{C}+\widetilde{S}_{D}
$$

$\widetilde{A}$ é a função de pertinência para a área transversal; $\widetilde{C}$ é a função de pertinência para a concentração; $\widetilde{u}$ é a função de pertinência para o campo de velocidade longitudinal; $\widetilde{E}$ é a função de pertinência para o coeficiente de dispersão longitudinal; $\widetilde{K}$ é a função de pertinência para o decaimento; $\widetilde{S}_{D}$ é a função de pertinência para o lançamento difuso. Condição de contorno para concentração, equação 6, e condição inicial para concentração, equação 7 .

$$
\begin{aligned}
\widetilde{C} & =\widetilde{C}_{0}(t) \text { em } \mathrm{x}=0 ; \frac{\partial \widetilde{C}}{\partial x}=\widetilde{C}_{1}(L) \mathrm{em} \mathrm{x}=\mathrm{L} \\
\widetilde{C} & =\widetilde{C}_{0}(x) \text { em } \mathrm{t}=0
\end{aligned}
$$

Em relação aos parâmetros referentes aos processos de transporte, definese:

$$
\widetilde{E}=0,05937 \frac{\widetilde{Q}}{\widetilde{S}_{0} B}
$$

$\widetilde{Q}$ é a vazão; $\widetilde{S}_{0}$ é a declividade do leito; e é a largura do canal. A solução deste conjunto de equações permite determinar as variáveis dependentes, na forma de função de pertinência. Estas funções são calculadas ao longo de trechos do rio para diferentes tempos. O campo de concentração é calculado pela equação fuzzy do transporte de massa. É importante notar que a solução da equação de transporte (5) produz as funções de pertinência de concentração no tempo e no espaço. Ou seja, há uma função de pertinência para a concentração, para cada seção do rio, em função do tempo. Isto implica que o modelo permite o acompanhamento da evolução do comportamento de uma nuvem poluente ao longo do rio, para diferentes instantes.

A questão é definir, no contexto da análise de risco, o papel de um campo de concentração, segundo as funções de pertinência. Como estas concentrações são calculadas a partir do lançamento de massas poluentes no corpo hídrico, de forma 
pontual ou difusa, sob as condições hidráulicas e hidrológicas do mesmo, é evidente que este campo de concentração representa a resposta do sistema hídrico para aquele lançamento. Esta resposta do sistema é a capacidade de um determinado corpo hídrico em receber cargas poluentes e se autodepurar ou não. Conclui-se assim, que este campo de concentração, obtido através da solução da equação de transporte de poluentes, desempenha um importante papel no cálculo e avaliação do risco.

A outra função de pertinência, também necessária para avaliar o risco de contaminação de um sistema hídrico, representa os níveis máximos de concentração permitidos, para diferentes substâncias. Esta função de pertinência representa, em suas características fuzzy, os limites máximos para as principais substâncias presentes nas diversas modalidades de lançamentos de esgotos, ou em um processo de drenagem de bacias com alto nível de atividades agrícolas. Esta função de pertinência é chamada de resistência e, uma vez definida, permite que o risco seja calculado.

Assim, $\widetilde{R}$ é a função de pertinência de resistência que representa os limites máximos de concentrações permitidos para determinados usos, em um sistema hídrico qualquer. E $\widetilde{C}$ a função de pertinência de concentração, calculada através do modelo matemático proposto, que representa a resposta do sistema receptor ao lançamento de determinadas substâncias. A margem de segurança $\tilde{M}$ deste corpo hídrico pode ser representada pela diferença entre a função de pertinência da resistência $\widetilde{R}$, e a função de pertinência da concentração $\widetilde{C}$, que representa a resposta aos possíveis lançamentos de cargas poluentes neste corpo hídrico, (GANOULIS, 1994). O índice de falha é definido pela equação 9, e o índice de confiabilidade pela equação 10.

$$
\begin{array}{r}
R_{f}=\frac{\int_{-\infty}^{0} \mu_{\widetilde{M}}(m) d m}{\int_{-\infty}^{\infty} \mu_{\widetilde{M}}(m) d m} \\
R_{c}=\frac{\int_{0}^{\infty} \mu_{\widetilde{M}}(m) d m}{\int_{-\infty}^{\infty} \mu_{\widetilde{M}}(m) d m}
\end{array}
$$




\subsection{Esquema Numérico para a Solução do Modelo de Transporte}

Tendo em vista a natureza da equação da difusão advectiva, o esquema numérico baseado no Método das Diferenças Finitas foi utilizado para resolver a equação de transporte. Este método permite a aproximação das derivadas parciais de forma explícita ou implícita. Nesta pesquisa, utilizou-se o esquema implícito, ou Método de Crank-Nicolson. A razão para tal escolha baseia-se no fato de que este tipo de discretização garante a estabilidade numérica no processo de solução (ANDERSON et al., 1984). É importante observar que tanto $R_{f}$ como $R_{c}$ são funções reais definidas no intervalo de $[0,1]$ e dependem, fundamentalmente, dos parâmetros hidráulicos/hidrológicos, da natureza, capacidade de escoamento do canal e do tempo.

As funções de pertinência de todos os parâmetros foram definidas como sendo funções triangulares apresentadas na forma, (CHAGAS, 2005),

$$
\begin{gathered}
\mu_{K}=\frac{k-k_{L}}{k_{m}-k_{L}}, \text { para } k_{L} \leq k \leq k_{m} \\
\mu_{K}=\frac{k-k_{u}}{k_{m}-k_{u}}, \text { para } k_{m} \leq k \leq k_{u}
\end{gathered}
$$

Os valores limites $k_{L}, k_{u}$ representam os valores dos parâmetros para os menores graus de pertinência e $k_{m}$ para o maior grau de pertinência. Para resolver as equações pertinentes ao modelo, um programa computacional foi desenvolvido em linguagem Fortran. Este programa permite avaliar o risco de contaminação em rios através da aplicação da Teoria Fuzzy, nos modelos de transporte de poluentes.

O processo de simulação, através do programa computacional desenvolvido, foi realizado com o objetivo de calcular o risco de contaminação de um corpo hídrico, sob a ação de agentes poluentes, considerando, apenas, lançamentos difusos. O programa calcula, inicialmente, o campo de concentração, em forma de funções de pertinência para várias seções do rio e diferentes tempos. Calculada a função de pertinência, e com o auxílio de uma função de pertinência de resistência, que representa o padrão de qualidade ambiental do sistema hídrico em estudo, pôde-se 
determinar uma distribuição para a função marginal de segurança ao longo do canal. A partir da função de pertinência marginal, a função risco pôde, finalmente, ser calculada.

\section{ANÁLISE DOS RESULTADOS}

Nas simulações, foi considerado um canal retangular com comprimento de $50.000 \mathrm{~m}$, discretizado em 50 trechos de $1000 \mathrm{~m}$ de comprimento, na direção longitudinal do rio. Com relação ao tempo, foram definidos 500 intervalos de tempo de $150 \mathrm{~s}$, perfazendo um tempo máximo de, aproximadamente, $21 \mathrm{~h}$. A vazão inicial do rio é de $50 \mathrm{~m}^{3} / \mathrm{s}$. Os valores da declividade do canal, da rugosidade e da vazão inicial são definidos de acordo com a simulação desejada.

Nas Figuras 1 e 2 verifica-se a distribuição da concentração para o lançamento de uma carga difusa, com $S_{D}=0,0005 \mathrm{mg} / \mathrm{m} / \mathrm{s}$, ao longo de todo o canal. $A$ concentração inicial é igual a zero, a rugosidade é igual a 0,05 e a declividade é igual a $0,0001 \mathrm{~m} / \mathrm{m}$. Entretanto, essas simulações são diferentes porque na primeira a substância considerada é conservativa, enquanto que na segunda a substância é não conservativa com decaimento igual a 0,0001/T. Como na Figura 1 não há decaimento, verifica-se que, para um tempo igual a $15 \mathrm{~h}$, o valor da concentração chega próximo de 30 mg/L. Já na Figura 2 a influência do decaimento faz com que a concentração, para o mesmo tempo, não ultrapasse $5 \mathrm{mg} / \mathrm{L}$.

FIGURA 1: Distribuição da concentração para o lançamento de uma carga difusa ao longo do canal para uma substância conservativa.

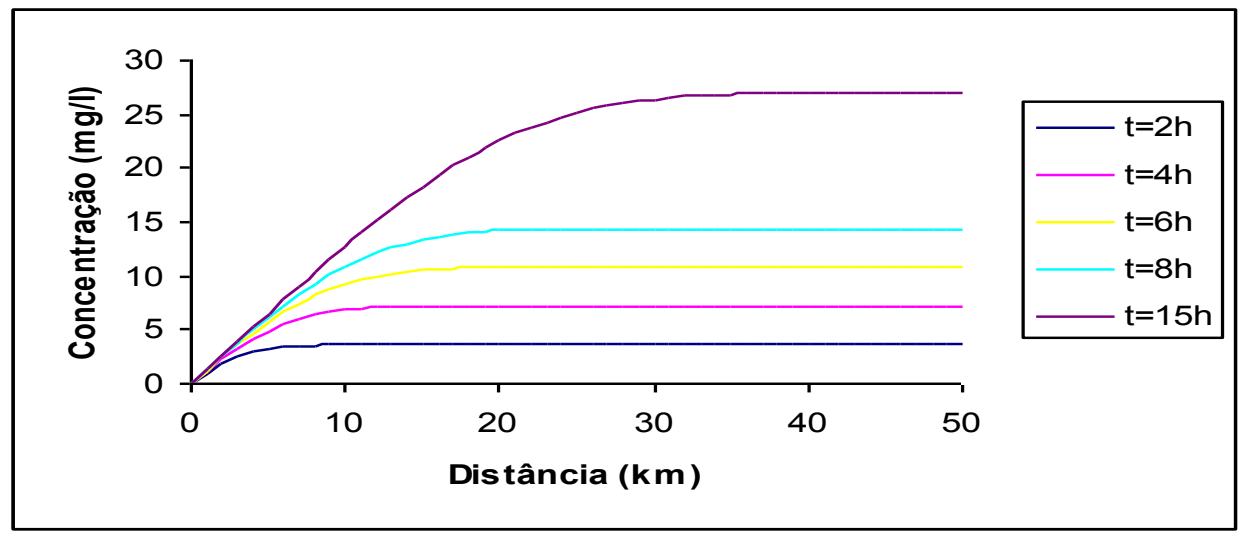


FIGURA 2: Distribuição da concentração para o lançamento de uma carga difusa ao longo do canal para uma substância não conservativa com $\mathrm{K}=0,0001 / \mathrm{T}$.

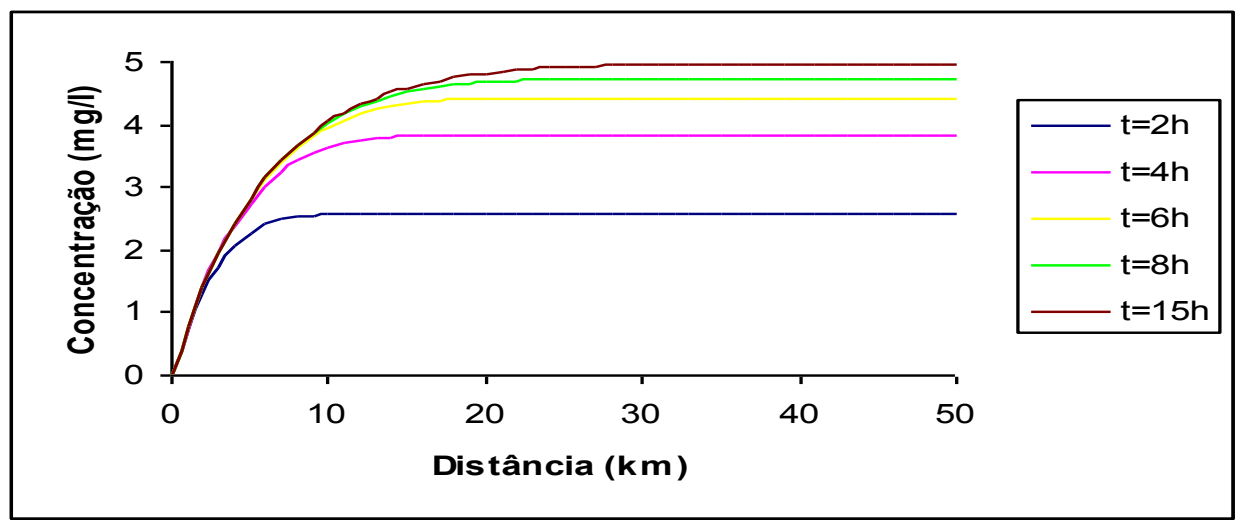

A Figura 2 ilustra ainda que para longos tempos de observação a concentração se estabiliza no valor $S_{D} / K$, onde $S_{D}$ representa a taxa de lançamento lateral, ou difuso, e $\mathrm{K}$ a taxa de decaimento. Este resultado está perfeitamente de acordo com os resultados obtidos a partir da solução analítica da equação da difusão advectiva, evidenciando, portanto, a coerência do modelo em estudo.

FIGURA 3: Distribuição da concentração para o lançamento de uma carga difusa num trecho parcial do canal, entre 5 e $20 \mathrm{~km}$, para uma rugosidade de 0,01 .

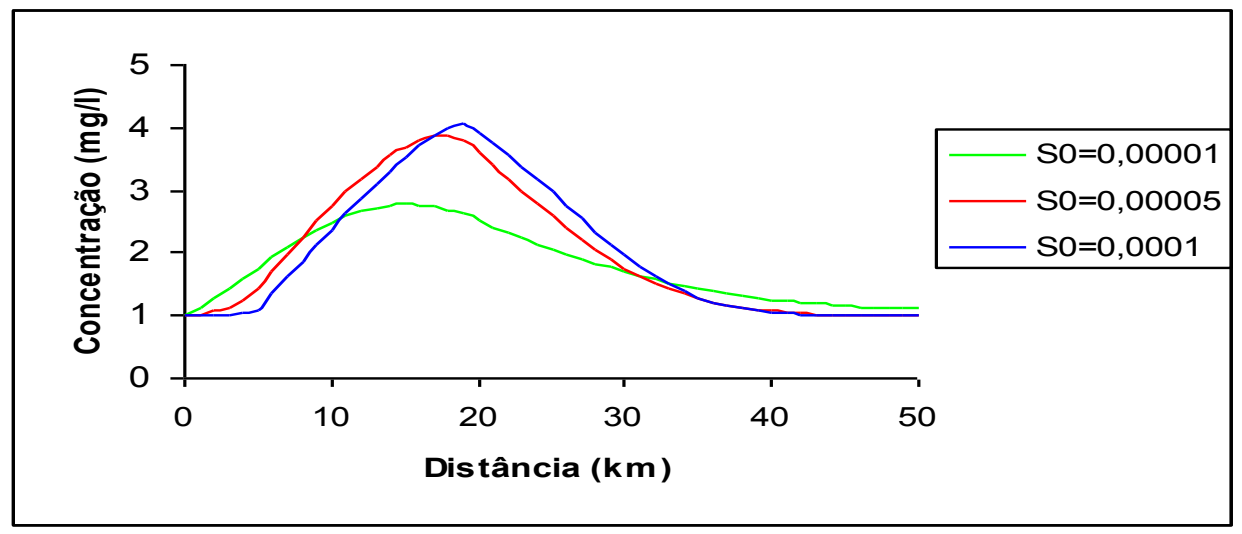

Na Figura 3 observa-se o cenário em que uma carga difusa é lançada em um trecho parcial do rio, com rugosidade igual a 0,01. Este trecho corresponde a um intervalo de $15 \mathrm{~km}$, compreendido entre 5 e $20 \mathrm{~km}$. Para pequenos valores de declividade, predomina-se o processo de difusão, no entanto, à medida que se aumenta estes valores, prevalece-se o processo advectivo. Por exemplo, para a declividade de $0,00001 \mathrm{~m} / \mathrm{m}$, a variância da concentração é maior que a variância para a declividade de $0,0001 \mathrm{~m} / \mathrm{m}$, indicando que a difusão no primeiro caso é mais intensa do que no segundo. Com isso, percebe-se que nos campos de velocidades 
menos intensos, mas ainda com a presença de turbulência, a difusão é mais significativa como processo de transporte. Para campos de velocidades maiores, prevalece-se o modelo advectivo.

Para testar a capacidade do modelo numérico fuzzy, simulou-se um exemplo simples de transporte de poluentes proposto por Dou et al. (1997). Nesta simulação, considerou-se uma condição de contorno para concentração de $100 \mathrm{mg} / \mathrm{l}$ e o comprimento do canal de $1525 \mathrm{~m}$. Na discretização, o canal é dividido em 100 trechos de 15,25 m de comprimento cada, com 1000 intervalos de tempo de 1 dia. Ainda, como dados de entrada foram utilizadas funções fuzzy triangulares para os parâmetros velocidade $(\mathrm{V})$, fator de ponderação para a dispersão $(\alpha)$, Coeficiente de dispersão $\left(E_{\mathrm{L}}\right)$, a saber, $V=[1,8 ; 2,4 ; 3,1] \mathrm{m} / \mathrm{s}, \alpha=[9,2 ; 15,3 ; 19,8] \mathrm{m}, \mathrm{E}_{\mathrm{L}}=[16,56 ; 36,72$; $61,38 \mathrm{~m}^{2} /$ dia. Verifica-se que as funções de pertinência triangulares são usadas para caracterizar os valores das entradas fuzzy nas simulações.

A partir da definição dos números fuzzy triangulares, com seus valores mínimo, médio e máximo, o programa gerou novos valores para as funções de pertinência, com cinco níveis diferentes 0 - $0.25-0.50-0.75-1$, totalizando 9 valores de concentração. As concentrações foram calculadas a uma distância de $1220 \mathrm{~m}$ da origem.

TABELA 1: Função de Pertinência de $C / C_{0}$ para $t=400$ e 800 dias.

\begin{tabular}{c|c|c|c|c|c|c|c|c|c} 
& $\mathbf{0}$ & $\mathbf{0 , 2 5}$ & $\mathbf{0 , 5 0}$ & $\mathbf{0 , 7 5}$ & $\mathbf{1}$ & $\mathbf{0 , 7 5}$ & $\mathbf{0 , 5}$ & $\mathbf{0 , 2 5}$ & $\mathbf{0}$ \\
\hline T=400d & 0 & 0,001 & 0,006 & 0,026 & 0,076 & 0,174 & 0,313 & 0,477 & 0,649 \\
\hline T=800d & 0,816 & 0,925 & 0,975 & 0,993 & 0,999 & 1 & 1 & 1 & 1 \\
\hline
\end{tabular}

A Figura 4 ilustra as funções de pertinência das concentrações, nos pontos apresentados na Tabela 1, para os tempos de 400 e 800 dias. 
FIGURA 4: Funções de pertinência, para as concentrações, obtidas pelo modelo desenvolvido, para os tempos de 400 e 800 dias.

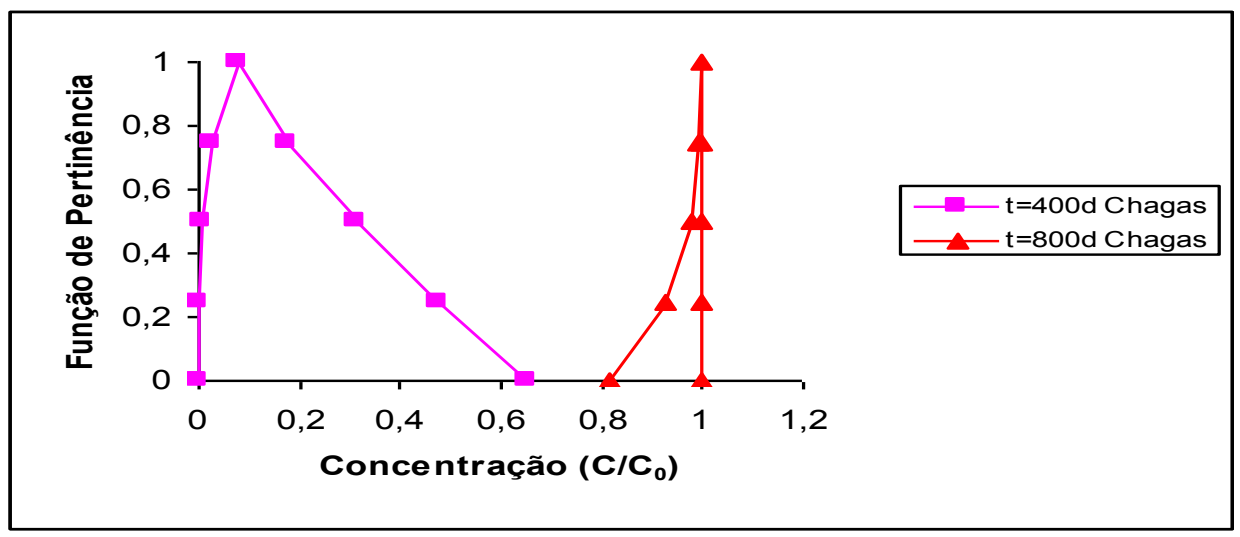

FIGURA 5: Comparação das funções de pertinência para o modelo desenvolvido e o modelo de Dou et al. (1997), para os tempos de 400 e 800 dias.

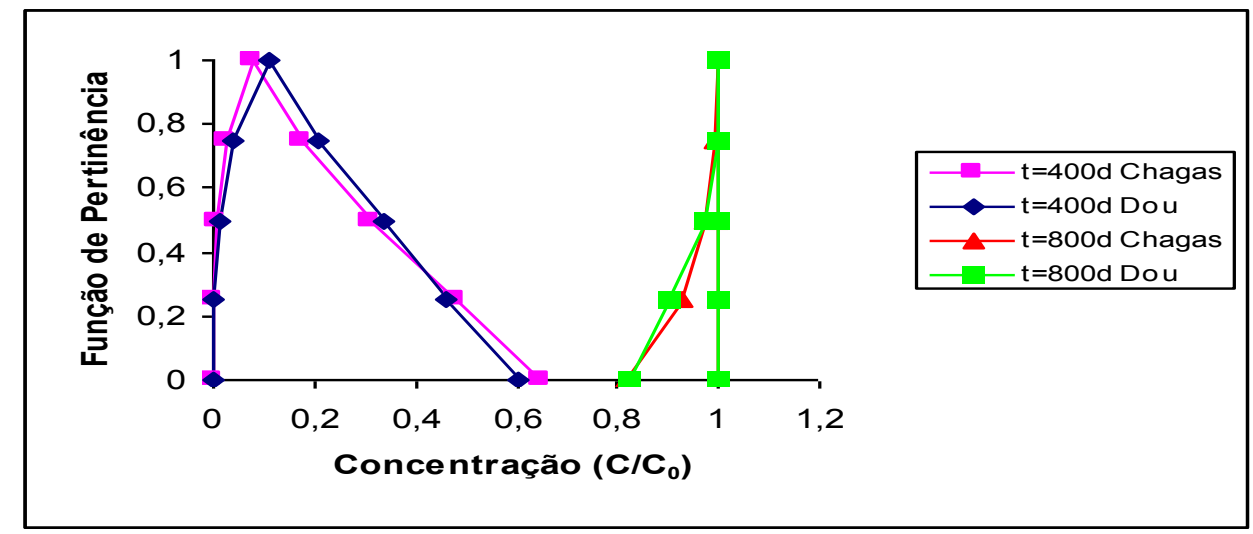

Na Figura 5, pode-se observar a comparação entre as funções de pertinência das concentrações obtidas pelo modelo desenvolvido, com as funções obtidas pela solução numérica do modelo de Dou et al. (1997). Verifica-se, com isto, que os valores do modelo desenvolvido coincidem quase que integralmente com os resultados de Dou et al. (1997), evidenciando a eficiência do modelo. 
FIGURA 6: Valores das concentrações fuzzy calculadas pelo modelo desenvolvido.

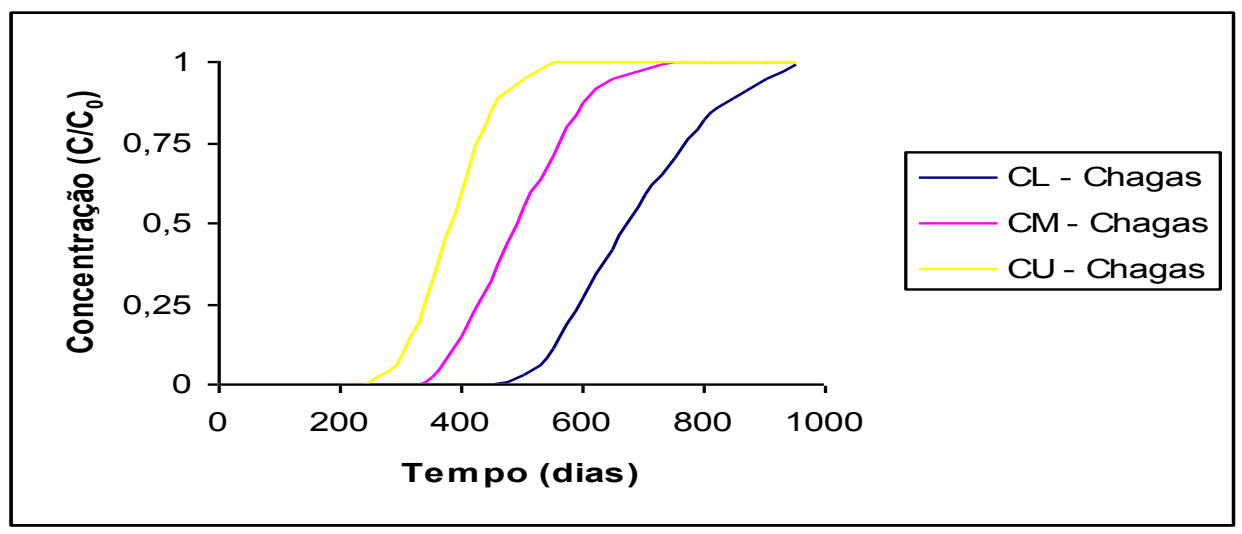

A Figura 6 ilustra os valores das concentrações calculadas, em cada nível, para diferentes tempos, entre os níveis 0 e 1. Têm-se, portanto, os valores: baixo $\left(\mathrm{C}_{\mathrm{L}}\right)$, médio $\left(C_{M}\right)$ e alto $\left(C_{U}\right)$ da concentração.

FIGURA 7: Comparação da concentração pelo modelo desenvolvido e pelo modelo de Dou et al. (1997).

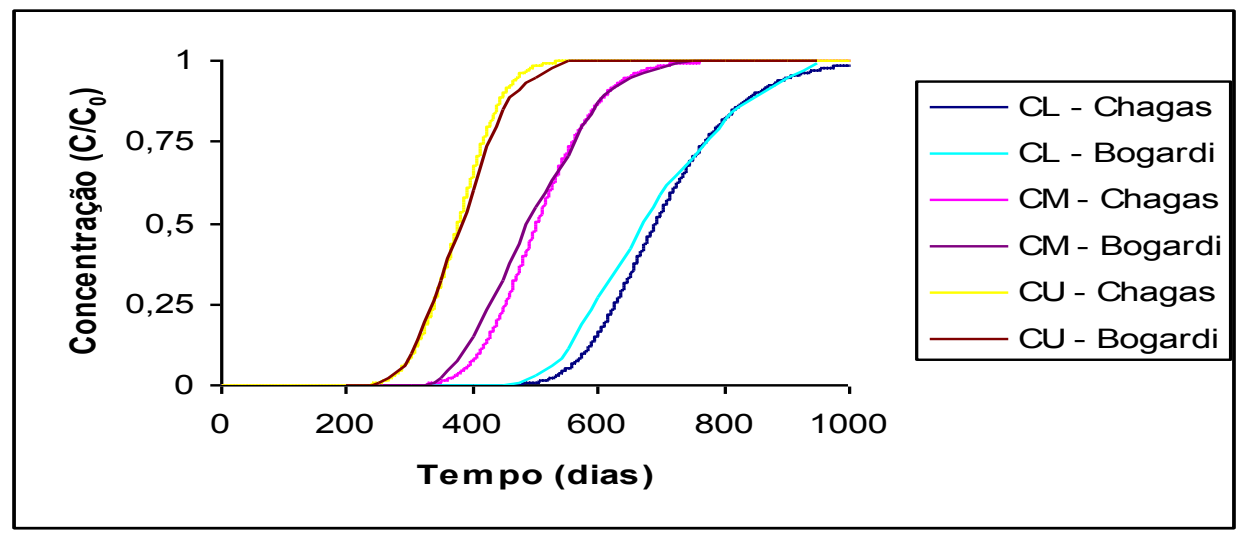

A Figura 7 ilustra a comparação entre os resultados das concentrações obtidas no modelo desenvolvido, com os resultados obtidos por Dou et al. (1997). Pelos resultados, conclui-se que a metodologia fuzzy proposta apresenta resultados que satisfazem plenamente aos objetivos da pesquisa. Uma vez comprovada a eficiência do modelo, foi avaliado o comportamento do risco de contaminação ambiental em um sistema fluvial. Nesta fase, foram feitas simulações mantendo as 
mesmas condições hidráulica e hidrológicas anteriores para determinar a função risco, no tempo e no espaço, após o lançamento de poluentes.

A Figura 8 ilustra o comportamento da função risco para uma situação onde há uma entrada difusa, ao longo do canal. A função de pertinência de resistência é definida por $A=[0,50,100] \mathrm{mg} / \mathrm{l}$, a concentração inicial é de $20 \mathrm{mg} / \mathrm{l}$ e há um lançamento difuso cuja função de pertinência é $A=[0,00001 ; 0,00005 ; 0,0001]$, com $\mu_{A}(0,00005)=1$. Verifica-se que o gráfico da função risco segue o mesmo padrão do perfil de concentração. Observa-se que a partir de $10 \mathrm{~km}$ as curvas têm um mesmo padrão de equidistância, ou seja, elas ficam paralelas. É importante notar que a inclinação da curva que representa a função risco no tempo depende da representação da função de pertinência para o lançamento difuso.

FIGURA 8: Comportamento do risco para um lançamento difuso ao longo do canal, com uma função de pertinência de resistência $A=[0,50,100] \mathrm{mg} / \mathrm{l}$.

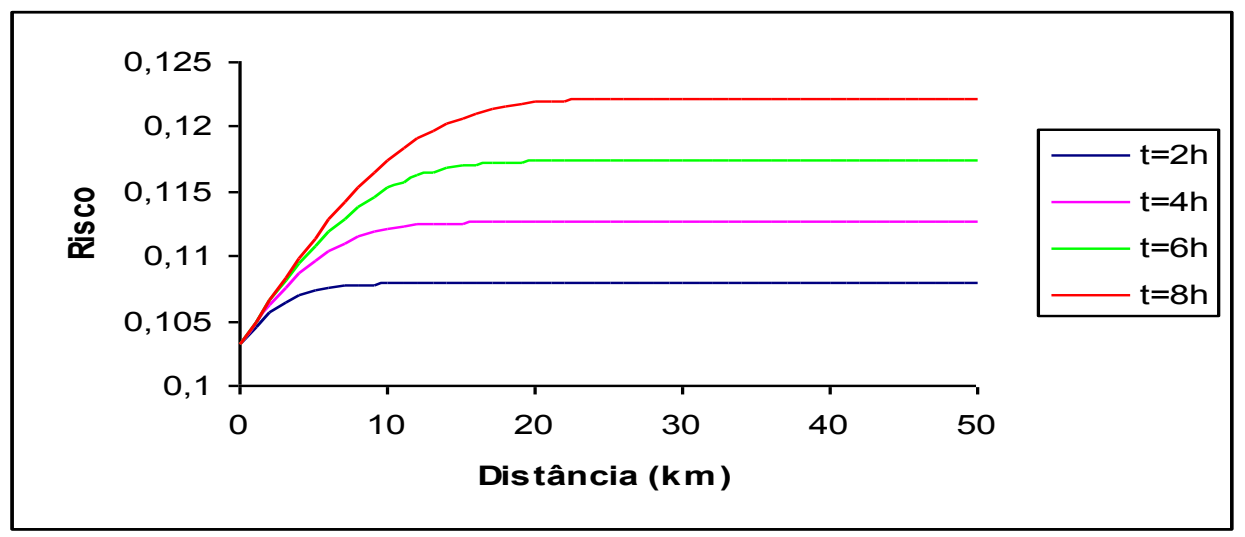

FIGURA 9: Comportamento do risco para um lançamento difuso ao longo do canal para uma observação em uma seção fixa do canal, a $10 \mathrm{~km}$ da origem.

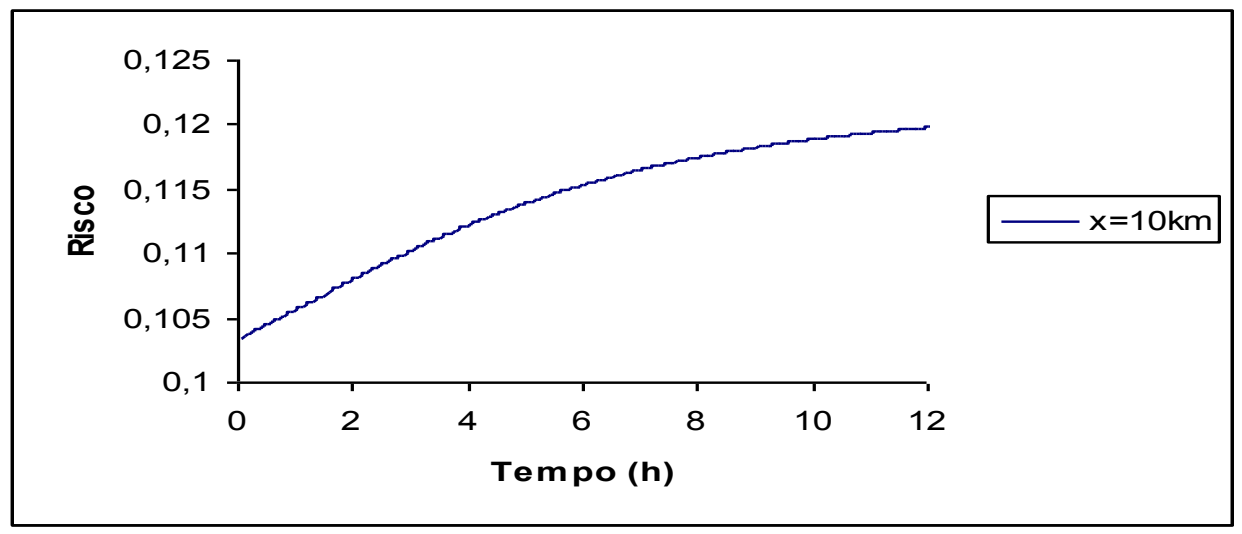


Na Figura 9 pode-se ver o comportamento do risco em uma seção de controle, a $10 \mathrm{~km}$ da origem, em função do tempo. É importante notar que a função risco tem um comportamento crescente, atingindo o valor de $12 \%$ para $t$ igual a 12 horas.

A Figura 10 ilustra a entrada de uma carga difusa, como no exemplo anterior, para substâncias não conservativas. A taxa de decaimento da substância considerada é $[0,000001 ; 0,000005 ; 0,00001]$. Neste caso, o risco cresce, mas não com as mesmas proporções anteriores. Observa-se que nas simulações para substâncias conservativas o risco teve seu pico em $12,3 \%$, enquanto que para substâncias com decaimento o pico do risco foi de 10,8\%, ambos para um tempo de 8 horas.

FIGURA 10: Comportamento do risco considerando uma substância não conservativa para lançamentos difusos.

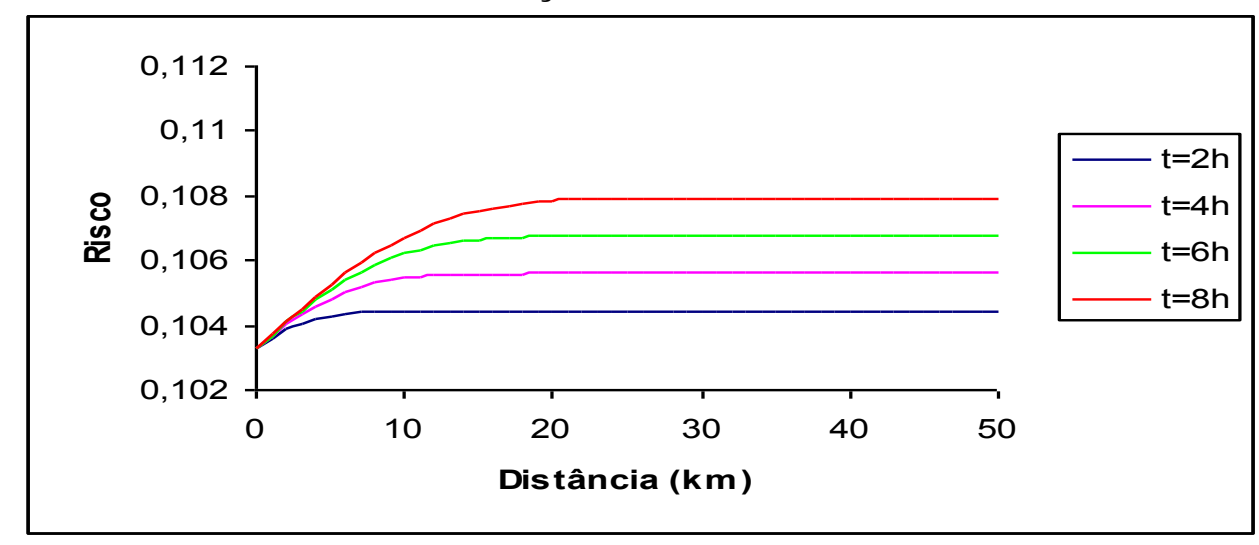

Estes resultados estão de acordo com a teoria dos processos de transporte. Neste cenário, a concentração da substância poluente tende para estabilidade cujo valor é $S_{D} / K$. $S_{D}$ é a taxa de lançamento difuso, enquanto que $K$ é a taxa de decaimento. 
FIGURA 11: Comportamento do risco com o tempo, considerando uma substância não conservativa para lançamentos difusos.

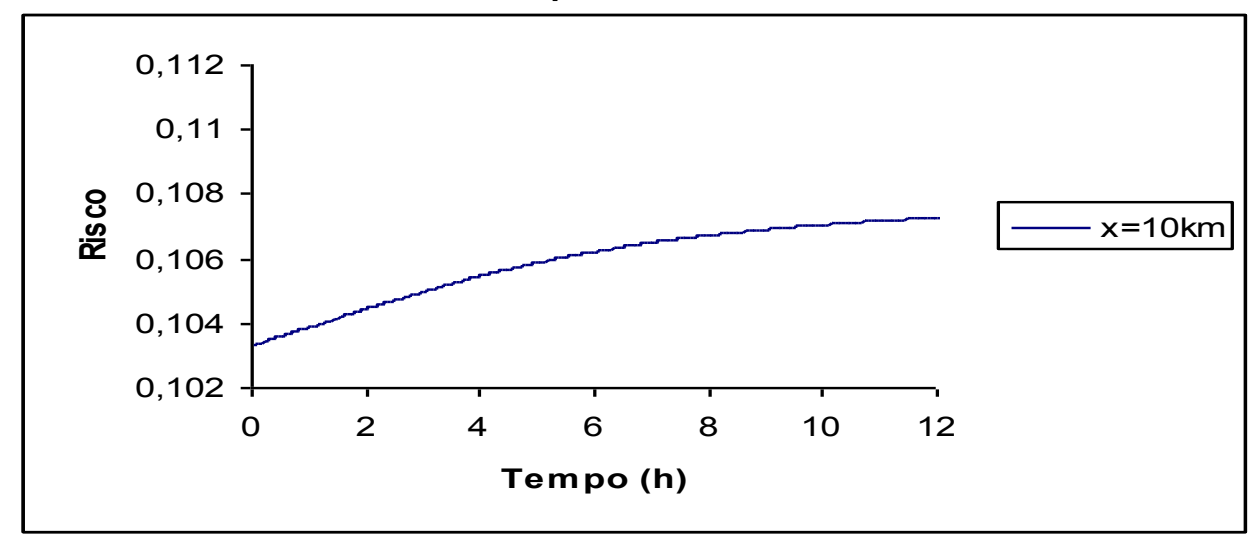

Nas Figuras 11, em que a observação é feita em $x$ igual a $10 \mathrm{~km}$, verifica-se que o comportamento crescente da curva de risco é mais acentuado para substâncias sem decaimento do que com decaimento, com os picos de $12 \%$ e $10,7 \%$, para o tempo de 12 horas, respectivamente.

FIGURA 12: Comportamento do risco com o tempo considerando uma substância não conservativa para lançamentos difusos.

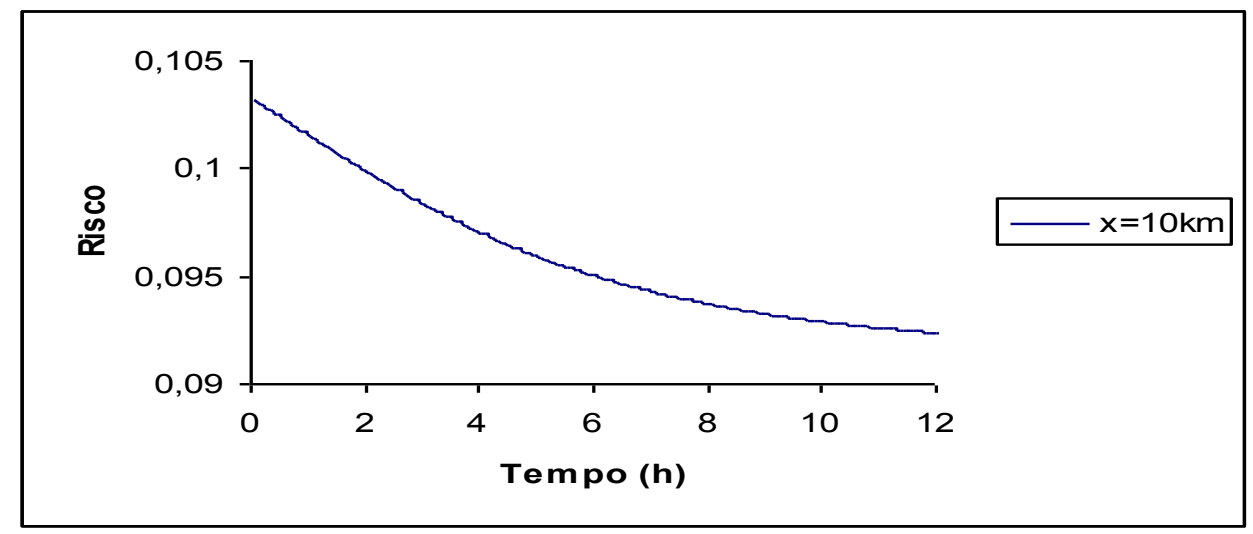

Na Figura 12, pode-se ver o risco numa seção de controle, a $10 \mathrm{~km}$ da origem, em função do tempo. Percebe-se, pela figura, que a queda nos valores do risco é contínua em função do padrão de decaimento da substância em análise. É importante notar que a capacidade de avaliar o risco, através desta metodologia é bastante satisfatória, tendo em vista que o modelo foi capaz de apresentar, em suas respostas, detalhes compatíveis com as observações de campo. 


\section{CONCLUSÕES}

Após a análise dos resultados obtidos pela aplicação do programa computacional desenvolvido, algumas conclusões podem ser formuladas:

Com relação aos resultados pertinentes à Teoria de Risco, pode-se concluir que o campo de risco, ao longo de um rio natural, tem estrutura de distribuição semelhante ao comportamento da concentração. Este fato permite ver que este campo é um funcional que tem como variáveis de controle funções relacionadas com os processos de transporte.

Pode-se ver também que, pela aplicação do modelo considerando lançamentos em rios, para um mesmo tipo de lançamento, rios com maiores vazões produzem campos de riscos menores. Enquanto que para alguns rios com vazões menores, o mesmo lançamento produziu um campo de risco que chegou próximo a seu estado de saturação. Isto permite concluir que nas questões de controle da conservação hidroambiental, os aspectos hidráulicos e hidrológicos desempenham papéis fundamentais.

Finalmente, uma análise global da metodologia apresentada permite verificar a grande versatilidade da Teoria Fuzzy, não somente na avaliação de risco como também no cálculo de campo de concentrações provenientes de modelos matemáticos fuzzy. E, a partir da pesquisa, nota-se que é possível fazer uma análise de risco com um restrito banco de dados, com a mesma eficiência de outras técnicas que demandam grandes quantidades de dados.

\section{REFERENCIAS}

ANDERSON, D. A.; TANNEHILL, J. C.; PLETCHER, R. H. Computational Fluid Mechanics and Heat Transfer. Hemisphere Publishing Corporation, 1984.

BOGARDI, I., DUCKSTEIN, L. The Fuzzy Logic paradigm of Risk Analysis. In: RISK-BASED DECISIONMAKING IN WATER RESOURCES X. Santa Barbara, California. Proceedings...New York, NY.: ASCE, p. 12-22, 2002.

CHAGAS, P. F. Perspectivas da Aplicação da Teoria Fuzzy para o cálculo de risco em sistemas hidrodinâmicos. Tese defendida no Departamento de Engenharia Hidráulica e Ambiental da 
Universidade Federal do Ceará como parte dos requisitos para obtenção do título de doutor em recursos hídricos, 2005.

CHAPRA, S. C.; RECKHOW, K. H. Engineering Approaches for Lake Management, v. 2. Mechanistic Modeling, Butterworth Publishers, 1983.

CHAPRA, S. C. Surface Water-Quality Modeling. New York: McGraw-Hill, 1997, 844p.

DOU, C.; WOLDT, W.; BOGARDI, I.; DAHAB, M. Numerical Solute Transport Simulation using Fuzzy Sets Approach. Journal of Contaminant Hydrology, n. 27, p. 107-126, 1997.

GANOULIS, J. G. Engineering Risk Analysis of Water Pollution: Probabilities and Fuzzy sets. VCH publishers Inc. - Weinheim; New York; Basel; Cambridge; Tokyo: 1994.

JAMES, A. An Introduction to WATER QUALITY MODELLING. 2nd Edition. By John Wiley \& Sons Ltd, 1993, 311p.

THOMANN, R. V.; MUELLER, J. A. Principles of Surface Water Quality Modelling and Control. Harper \& Row, Publishers, New York, 1987. 